RAE-IC, Revista de la Asociación Española de Investigación de la Comunicación vol. 8, núm. 15 (2021), 242-266 ISSN 2341-2690

Recibido el 30 de octubre de 2020 DOI: https://doi.org/10.24137/raeic.8.15.11 Aceptado el 22 de diciembre de 2020

\title{
Una metainvestigación cualitativa para (re)construir el campo: objetivos, perspectivas teóricas y métodos para la investigación en comunicación
}

A qualitative metaresearch to (re)build the field: typology of objectives, theoretical perspectives and methods for communication research

Gómez-Diago, Gloria

Universidad Rey Juan Carlos (URJC)

gloria.gomez.diago@urjc.es

Forma de citar este artículo:

Gómez-Diago, G. (2021). Una metainvestigación cualitativa para (re)construir el campo: objetivos, perspectivas teóricas y métodos para la investigación en comunicación. $R A E-I C$, Revista de la Asociación Española de Investigación de la Comunicación, 8(15), 242-266. https://doi.org/10.24137/raeic.8.15.11

\section{Resumen:}

Mientras la comunicación, especialmente la comunicación digital, es de interés para campos consolidados como la sociología o las ciencias políticas y para campos de creación más reciente como la biblioteconomía, el campo de la investigación en comunicación funciona como una subdisciplina amnésica sin ambición disciplinar (Pooley, 2020). Con el objetivo de contribuir a (re) construir intelectualmente un campo que puede ser útil a la sociedad, se aporta una tipología de objetivos, de perspectivas teóricas y de métodos de investigación, generada a partir de una destilación del 
volumen 2 del Rethinking Communication (1989), "Paradigm Exemplars", volumen especializado en metainvestigación en comunicación y considerado como una continuación del volumen especial del Journal of Communication, "Ferment in the Field", publicado en 1983 (Surlin, 1991). Las herramientas conceptuales y procedimentales traídas y ordenadas en aquellas orientadas a estudiar relaciones entre personas y las orientadas a estudiar relaciones entre personas y medios de comunicación, son útiles en un contexto en el que las relaciones humanas y las relaciones entre las personas y los medios de comunicación siguen articulando las sociedades. Es necesario revisar y redefinir las maneras de abordarlas para ampliar el alcance de un campo limitado desde hace décadas, fundamentalmente, al estudio de los contenidos de los medios de comunicación (Cáceres y Caffarel, 1992; MartínezNicolás y Saperas, 2011, 2016; Anderson y Middelton, 2015), mediante un análisis de contenido que Krippendorff (2017) recomienda abandonar.

Palabras clave: comunicación, epistemología, historia de la investigación en comunicación, metainvestigación de las ideas, metodología, métodos de investigación, teoría de la comunicación, teorías de la comunicación, “Paradigm Exemplars" (1989).

\section{Abstract:}

As long as communication, especially digital communication, is of interest for consolidated fields such as sociology or political sciences and for fields of more recent creation such as librarianship, the field of communication research functions as an amnesic sub-discipline without disciplinary ambition (Pooley, 2020). In order to contribute to intellectually (re) constructing a field that can be useful to society, we are providing a typology of objectives, theoretical perspectives and methods generated from a distillation of the volume 2 of Rethinking Communication (1989), "Paradigm Exemplars", a volume specialized in meta-research in communication and considered as a continuation of the special volume of the Journal of Communication, "Ferment in the Field", published in 1983 (Surlin, 1991). The conceptual and procedural devices brought and grouped into those aimed at studying relationships between people and those aimed at studying relationships between people and media, are useful in a moment in 
which human relationships and relationships between people and media continue articulating societies. It is necessary to review and redefine the ways of approaching them to further the scope of communication research, limited for decades, fundamentally, to the analysis of media content (Cáceres and Caffarel, 1992; MartínezNicolás and Saperas, 2011, 2016; Anderson and Middelton, 2015) through a content analysis that Krippendorff (2017) recommends to leave.

Keywords: communication, communication theory, epistemology, history of communication research, methodology, metaresearch, metaresearch of ideas, methods of research, "Paradigm Exemplars" (1989), theories of communication.

\section{INTRODUCCIÓN}

La producción investigadora sobre comunicación se ha acelerado en los últimos años, viéndose facilitada esta aceleración por la continua aparición de revistas académicas que han venido generando una hiperespecialización (Martínez-Nicolás et al., 2019) en un campo científico falto de consolidación intelectual (Peters, 1986; Lacasa, 2017), amnésico en lo referido a su historia (Pooley, 2020), carente de propuestas teóricas (Martínez-Nicolás y Saperas, 2011, 2016; Neuman y Guggenheimm, 2011; Bryant y Miron, 2004, Pacanowsky, 1989) y de revisiones del andamiaje intelectual en el que se ha venido sustentando. Aun cuando se ha venido desarrollando también de manera exponencial investigación acerca de la investigación en comunicación (Martínez-Nicolás, 2020), se ha prestado poca atención a los objetivos y a los métodos de investigación que han venido definiendo este campo.

Buena parte de los trabajos de metainvestigación en comunicación atienden a características formales de la producción científica relacionadas con el tipo de autoría, y a los llamados "objetos de estudio", que suelen corresponderse con tipos de comunicación según su finalidad (publicidad, periodismo) y en un nivel de análisis más profundo se corresponden con los procesos que intervienen en la comunicación; siendo también frecuente la metainvestigación que identifica las "temáticas" más tratadas en 
el campo (Günter y Domahidi, 2017). Al no poder basarse la institucionalización intelectual del campo en ocuparse de algo de interés internacional como la comunicación (Peters, 2008, p. 143), es difícil construir una epistemología a partir de esa metainvestigación.

Otra línea de metainvestigación, que es en la que se sitúa este trabajo, se ocupa de profundizar en el andamiaje intelectual en el que se sustenta este campo científico, identificando perspectivas de investigación e intereses de conocimiento (MartínezNicolás, 2001, 2006, 2007), identificando las corrientes teóricas utilizadas en España (García Jiménez, 2007), analizando la presencia de los diferentes paradigmas en la investigación (Bermejo-Berros, 2014; Potter; Copper y Dupagne, 1993), evaluando la función de la epistemología y de las teorías en la producción (Byrant y Miron, 2004), o identificando consecuencias epistemológicas de la investigación actual (MartínezNicolás, Saperas, Humanes, 2019).

Aún y cuando, como sucedía cuando Berelson (1959) expresaba su preocupación por la carencia intelectual del campo, hay otras formas de florecimiento ${ }^{1}$ en el campo, es necesario nutrirlo epistemológicamente. A este objetivo puede contribuir una metainvestigación que se ocupe de los métodos de investigación, vinculándolos a las teorías y a los objetivos de investigación, una metainvestigación que genere una "caja de herramientas" que puedan ser actualizadas y/o usadas para (re) construir intelectualmente un campo que sufre la "dependencia funcional" que afecta a los campos científicos que teniendo escaso prestigio, dependen de normas de campos más prestigiosos (Withley, 2000, pp. 268-282; en Pooley, 2017, p. 330).

Una metainvestigación de las ideas que han venido motivando y que motivan la investigación en comunicación posibilita (re) dibujar herramientas conceptuales y

\footnotetext{
${ }^{1}$ Se ha comprobado un aumento en el número de artículos publicados en la década de 1990 (Martínez-Nicolás y Saperas, 2011), así como un incremento en la presencia de autores españoles en revistas extranjeras durante 1994-2009 (Masip, 2011; MartínezNicolás, 2014) y en revistas indexadas en el Social Sciences Citation Index (Contreras et al. 2011), un incremento en la publicación libros sobre comunicación en los últimos 25 años (Soriano, 2017, p. 47), un aumento en el número de proyectos financiados entre 2012 y 2013 (Caffarel-Serra, Ortega-Mohedano, Gaitán-Moya, 2017, p. 22) o un aumento en el número de tesis doctorales defendidas: casi mil en el período 2007- 2013 (Escobar y Martín-Algarra, 2017).
} 
procedimentales para (re) articular un campo científico que puede ser de utilidad para la sociedad.

La mayor parte de la metainvestigación en comunicación que atiende a los métodos de investigación pone atención a si los trabajos analizados utilizan métodos de investigación cuantitativos, cualitativos o mixtos.

Es fundamental prestar atención a las perspectivas, a las teorías, a los conceptos y a los procedimientos que han venido definiendo y que definen al campo científico de la comunicación. En este esfuerzo se sitúa este trabajo, ubicado en una línea de investigación que denominamos metainvestigación de las ideas, centrada en esta oportunidad en identificar objetivos y métodos de investigación, elementos indisolubles en la actividad científica (Comte, en Bordieu; Chamboredon y Passeron, 2002, p. 11), asociándolos a las perspectivas teóricas desde las que se utilizaron, pues la aproximación teórica al objeto y no el objeto en sí mismo, inspira la construcción del método de investigación (Bericat, 1998, p. 97, 98).

Este trabajo es un paso en la tarea de ubicar y ordenar herramientas conceptuales y procedimentales para (re) articular el campo científico de la comunicación. A partir de la revisión sistemática de los artículos del volumen 2 de Rethinking Communication, titulado "Paradigm Exemplars" (1989), se propone una tipología de objetivos, perspectivas teóricas y métodos de investigación centrados en estudiar las relaciones humanas y las relaciones entre las personas y los medios de comunicación.

\section{MÉTODO DE INVESTIGACIÓN UTILIZADO Y CORPUS ANALIZADO}

Este trabajo presenta una tipología de objetivos, perspectivas teóricas y métodos de investigación para investigar acerca de las relaciones entre las personas y acerca las relaciones entre las personas y los medios de comunicación, generada a partir de la destilación de los treinta artículos del volumen 2 del Rethinking of Communication, "Paradigms Exemplars", editado por Dervin, Grossberg, O' Keefe y Wartella y publicado en 1989 por Sage y por la International Association of Communication (ICA). Este volumen es de acuerdo con Surlin (1991) una extensión del diálogo iniciado con el 
volumen especial del Journal of Communication "Ferment in the Field" (1983), al que Nordenstrend señaló (2004, p. 7) como el lugar para buscar un "alma" que guiase a la investigación en comunicación. Se trata de un volumen fundamental principalmente por dos motivos. En primer lugar, porque recoge una diversidad de perspectivas y de problemas de investigación que motivan el campo de la comunicación ${ }^{2}$. En este sentido, tanto el volumen II como el volumen I del Rethinking Communication, titulado "Paradigm issues", se plantearon como resultado del Congreso de la International Communication Association (ICA), "Beyond Polemics: Paradigm Dialogues"3 (1985) para generar diálogo entre perspectivas en el campo de la comunicación. Los editores del volumen pidieron a los autores que explicasen el modelo analítico que utilizaban en sus trabajos, y que proporcionasen un ejemplo de aplicación del modelo.

En segundo lugar, este volumen es fundamental porque los autores de los artículos que lo componen son referentes y han ido definiendo el campo con sus perspectivas y prácticas, como demuestra el elevado número de citas que tienen sus trabajos ${ }^{4}$.

\section{OBJETIVOS, PERSPECTIVAS TEÓRICAS Y MÉTODOS DE INVESTIGACIÓN PARA ESTUDIAR RELACIONES ENTRE PERSONAS Y RELACIONES ENTRE PERSONAS Y MEDIOS DE COMUNICACIÓN}

A continuación, se presentan algunos objetivos, perspectivas teóricas y métodos de investigación a partir de los que articular investigación en comunicación centrada en las relaciones entre personas y en las relaciones entre personas y medios de comunicación.

\footnotetext{
2 Si bien en el volumen "Paradigm exemplars" (1989) se aportan diferentes maneras de abordar la investigación en comunicación, solo seis de los 34 autores del volumen pertenecían a departamentos que no fuesen de comunicación: Frankel y Beckman (Medicina interna), Goodwin (Lingüística aplicada), Graber (Ciencia política), Mosco (Sociología), Daryl Slack (Humanidades). En este sentido, este volumen refleja una variedad de perspectivas dentro del campo, situación que ha cambiado con respecto a la actualidad, en la que desde campos diferentes se investiga acerca de la comunicación (Pooley, 2020).

${ }^{3}$ El congreso de la ICA "Beyond Polemics: Paradigm Dialogues" (1985) pretendía "(...) ir más allá de polémicas en el sentido de transcender diferencias superficiales y polaridades y permitirnos obtener un mayor entendimiento de las maneras fundamentales en las que nos diferenciamos y en las maneras en las que algunas de estas diferencias pueden no tanto diferenciarnos sino enriquecernos" (Dervin, Grossberg, O ' Keefe y Wartella, 1989, p. 22).

${ }_{4}^{4} \mathrm{~A}$ pesar de que la mayor parte de autores del volumen carece de perfil en Google scholar, destacan los índices de Wasko (h-index 34; i10-index 57); Turow (h-index 42, i-10 index: 81); Daryl Slack (h-index18, i-10 index 25); Tracy (h-index: 49, i-10 index: 83), Thorson (h-index:58, i-10 index: 139) o Murdock (h-index:55; i-10 index: 139). Buscando a los demás autores del volumen en Google Scholar, se encuentra que sus trabajos tienen un elevado número de citas. En este sentido, a fecha de 3 de septiembre, 2020, Edna Rogers tiene trabajos como "Analysis of relational communication in dyads: New measurement procedures" con 470 citas y Charles Goodwing, tiene un trabajo titulado "Conversation analysis" con 1662 citas.
} 


\subsection{RELACIONES ENTRE PERSONAS}

Se plantean objetivos, perspectivas teóricas y métodos de investigación para el estudio de las relaciones humanas en el ámbito personal y en el ámbito profesional.

\subsubsection{Relaciones en el ámbito personal}

En este apartado se incluyen objetivos, perspectivas teóricas y métodos de investigación que abordan la comunicación como una competencia y trabajos que estudian las relaciones desde una perspectiva comunicativa. Esta perspectiva comunicativa tiene en cuenta diferentes elementos que dan forma a la comunicación, entendiendo a esta última como una variable dependiente en lugar de como variable independiente. Una concepción de la comunicación como variable independiente ha motivado la mayor parte de la investigación en comunicación, usualmente orientada a evaluar los efectos de la comunicación, mediante trabajos que sitúan los elementos fuente, mensaje, canal y/o receptor como variables independientes que se modifican para evaluar las consecuencias de su manipulación en los efectos de comunicación (Rogers y Kincaid, 1981, p. 34).

En cuanto al estudio de las competencias comunicativas, Burleson (1989) evaluó la capacidad persuasiva de los niños y de los adolescentes, planteando esta habilidad como dependiente de la habilidad socio-cognitiva, utilizando dos índices para evaluarla, 1) complejidad cognitiva interpersonal y 2) perspectiva social y relacionándolos con la edad. El investigador presentaba a los niños situaciones para que desarrollasen un mensaje que él puntuaba en función de su grado de adaptabilidad, de si se tenían en cuenta las motivaciones del persuadido.

También interesada en las competencias comunicativas y considerando que el propósito de la investigación conversacional debe ser clarificar las elecciones de prácticas comunicativas y guiar en las elecciones necesarias para conseguir determinados objetivos, Tracy (1989) propuso el "experimento naturalista", donde los participantes escribían lo que responderían a situaciones estandarizadas grabadas. 
Entendiendo la comunicación como competencia, pero también como relación, desde el paradigma que denominó Network Paradigm, Yum (1989) comprobó si había diferencias estructurales entre las redes de grupos étnicos y las redes de inmigrantes y si estas diferencias afectaban a la información que tenían disponible y a sus actitudes hacia otros grupos étnicos. Entre 1978 y 1979, la investigadora encuestó a cinco grupos étnicos de Hawáii y estudió sus redes de comunicación a partir índices orientados a explicar el nivel de información de los inmigrantes en función de su conocimiento de la agencia social que les proveía servicios en Honolulu.

Diferentes objetivos, perspectivas teóricas y métodos de investigación estudian las relaciones poniendo atención en las conversaciones. Desde una perspectiva que entiende también la comunicación como competencia e interesado en conocer cómo las personas construyen el orden social a través del lenguaje, Goodwin (1989) analizó los turnos en el habla mediante métodos sensibles a la interacción colaborativa como grabaciones de conversaciones en entornos naturales. De acuerdo con Goodwin, analizar los turnos en el habla y las conversaciones permite estudiar competencias sociales como la habilidad para construir significado, para crear un mundo personal coherente, para producir lenguaje y para construir orden social.

Respecto de cómo plantear el análisis conversacional, Jacobs y Jackson (1989) propusieron trabajar desde una perspectiva inductiva en la que los conceptos y las categorías resultantes emergiesen de una observación detallada.

También desde una perspectiva que entiende la comunicación como competencia, Kellermann y LIM (1989) estudiaron conversaciones para saber cómo comienzan y evolucionan las relaciones en función de ese comienzo. Utilizaron los MOPs (Memory Organization Packets), estructuras cognitivas que organizan secuencias para lograr determinados propósitos en una situación conversacional.

Partiendo de la Action Assemble Theory, teoría centrada en explicar y en predecir la conducta humana y poniendo atención a la producción de conductas comunicativas verbales y no verbales, de conductas pensadas y automáticas, rutinarias y creativas; Green (1989) hizo experimentos centrados en las consecuencias no verbales del engaño. 
Interesado en la relación entre culturas y conductas comunicativas y entendiendo que los recursos para generar acciones coordinadas varían según los grupos sociales y que esa variabilidad debe ser considerada al explicar la comunicación humana, Philipsen (1989) propuso investigar desde una perspectiva etnográfica acerca de cómo las personas comparten significados. El investigador planteó la necesidad de desarrollar un modelo de descubrimiento para entender sistemas comunicativos diferentes, para conocer la naturaleza y el alcance de la variación cultural en la conducta comunicativa, para descubrir, describir y analizar comparativamente modelos de comunicación particulares. Philipsen (1989) buscaba que las etnografías no estuviesen guiadas por un modelo sino que proveyesen datos para redefinirlo, de manera que su desarrollo se fundamentase empíricamente, generando el modelo teórico.

Planalp (1989), interesada en la representación y en la utilización del conocimiento relacional, esto es en cómo se llega a él, en cómo se usa este conocimiento y en cómo éste cambia en respuesta a eventos, analizó las relaciones desde una perspectiva social cognitiva. Desde esta perspectiva se considera que la información se selecciona y se usa para guiar la conducta; que las personas desarrollan estructuras y cuerpos de conocimiento que guían los procesos cognitivos, produciéndose una influencia entre procesos cognitivos, estructuras de conocimiento y conducta.

De acuerdo con Planalp, se necesita una perspectiva cognitiva para estudiar las relaciones porque la información acerca de las relaciones se selecciona de la interacción o de la conducta y se integra con otra información y con conocimiento previo, usándose esta información para hacer inferencias, para almacenarla y recuperarla desde la memoria y utilizarla en siguientes interacciones.

En el contexto familiar, Rogers (1989) utilizó la perspectiva relacional para estudiar matrimonios, realizándoles entrevistas y encuestas. De acuerdo con la investigadora, la perspectiva relacional se basa en una epistemología centrada en estudiar los modelos de interacción en las relaciones.

También interesada en las relaciones matrimoniales, específicamente en el papel de la comunicación en los matrimonios como causa de felicidad, desde la perspectiva del 
conocimiento social (social knowledge perspective), que estudia el uso de la comunicación interpersonal para hacer inferencias acerca de las personas, Sillars (1989) hizo cuestionarios a mujeres casadas y analizó conversaciones entre matrimonios.

\subsubsection{Relaciones en el ámbito profesional}

Los objetivos, las perspectivas teóricas y los métodos de investigación expresados a continuación permiten visualizar el alcance de un campo de la investigación en comunicación que puede (re) orientarse a mejorar los contextos profesionales en diferentes dimensiones.

Mosco (1989), desde una perspectiva sociológica crítica, identificó cuatro procesos que caracterizan la actividad laboral: 1) Dominación/hegemonía, 2) Contradicción, 3) Oposición y 4) Transformación. Interesado en cómo las tecnologías afectaban a las relaciones entre los trabajadores, el investigador hizo entrevistas abiertas a trabajadores de una empresa de venta por teléfono.

Frankel y Beckman (1989) tenían como objetivo que los pacientes siguiesen las prescripciones de los médicos. Considerando la enfermedad como una percepción individual y como una respuesta social a cambios en el estado de salud y entendiendo al individuo en términos relacionales, en continua interacción con su entorno, realizaron análisis conversacional.

Siguiendo en el entorno profesional, estudiar las "culturas organizacionales" como la totalidad de las experiencias vividas en las organizaciones, poniendo atención en los procesos y en las prácticas mediante las cuales sus miembros dan sentido a su experiencia, es lo que propuso Pacanowsky (1989) como objetivo para la investigación en comunicación. Desde una perspectiva interpretativa que sitúa entre la perspectiva funcionalista y la perspectiva crítica, y que entiende que busca dar sentido al mundo entrando en diálogo con otros, dando sentido a los sentidos realizados por otros, Pacanowsky (1989) utilizó el docudrama, combinando ficción y revisiones de literatura, con un estilo confesional en primera persona para describir experiencias en conferencias y en convenciones académicas. 
Las organizaciones pueden también ser estudiadas desde una perspectiva de red (Stohl, 1989), analizando las propiedades de su estructura de comunicación, como el grado de interconexión y de agrupación de los enlaces, el grado complejidad de las relaciones o el grado de centralidad o proximidad en la red. Este tipo de análisis permite, según la investigadora, incorporar y extender las nociones convencionales de estructura y centrarse en la complejidad y en el dinamismo de las relaciones sociales. En lugar de buscar respuestas en las características individuales, en las historias o en las instituciones, se buscan respuestas en las características de las relaciones.

Desde la Teoría de Red, Stohl (1989) planteó "círculos de calidad" integrados por trabajadores que ayudaban a compañeros a resolver dudas y realizó entrevistas a estos trabajadores para conocer los cambios que los círculos introdujeron en el funcionamiento de la organización, además de encuestas para conocer las características de la comunicación que establecían con sus compañeros.

También interesado en el funcionamiento de la comunicación en las instituciones, Mc Phee (1989), considerando que la estructura y la interacción deben entenderse fusionadas, entrevistó a integrantes de organizaciones desde la Teoría de la Estructuración (Giddens, Bourdieu, Bhaskar, o Touraine), teoría que atiende al posicionamiento de los individuos como sujetos en las instituciones, a la configuración de sus perspectivas, poderes, responsabilidades, o carreras así como a las funciones representadas y asumidas por ellos y por otros.

A continuación, se incluye una tabla en la que se sitúan objetivos, perspectivas teóricas y métodos de investigación referidos a relaciones entre personas en el entorno personal y en el entorno profesional. 
Tabla 1. Objetivos, perspectivas teóricas y métodos de investigación para estudiar las relaciones humanas en el entorno personal y profesional en "Paradigm exemplars"

(1989).

\begin{tabular}{|c|c|}
\hline \multicolumn{2}{|r|}{ Relaciones entre personas } \\
\hline Objetivos de investigación & Perspectivas y métodos de investigación \\
\hline \multicolumn{2}{|r|}{ Entorno personal } \\
\hline \multicolumn{2}{|c|}{ Comunicación como competencia } \\
\hline $\begin{array}{l}\text { Evaluar la capacidad persuasiva } \\
\text { de niños, adolescentes y adultos }\end{array}$ & $\begin{array}{c}\text { Presentación de situaciones hipotéticas a niños para que } \\
\text { desarrollasen un mensaje persuasivo. Análisis conversacional } \\
\text { y análisis del pedido de favores. Exposición a personas a } \\
\text { conversaciones para que expresasen como conseguirían un } \\
\text { propósito determinado. }\end{array}$ \\
\hline $\begin{array}{l}\text { Conocer el grado de integración } \\
\text { de un grupo social }\end{array}$ & $\begin{array}{c}\text { Análisis conversacional. Análisis de turnos del habla. Uso del } \\
\text { experimento naturalista para analizar respuestas } \\
\text { proporcionadas libremente en relación a situaciones } \\
\text { estandarizadas. }\end{array}$ \\
\hline \multicolumn{2}{|r|}{ Comunicación como relación } \\
\hline $\begin{array}{l}\text { Analizar cómo se generan las } \\
\text { relaciones }\end{array}$ & $\begin{array}{l}\text { Perspectiva que combina el uso de las MOPs o Memory } \\
\text { Organization Packets }{ }^{5} \text { con el análisis del contexto de la } \\
\text { conversación, utilizando encuestas y observación. }\end{array}$ \\
\hline $\begin{array}{l}\text { Conocer cómo funciona la } \\
\text { comunicación en matrimonios }\end{array}$ & $\begin{array}{l}\text { Desde la perspectiva relacional y desde la perspectiva social } \\
\text { del conocimiento, entrevistas y encuestas a matrimonios. }\end{array}$ \\
\hline \multicolumn{2}{|r|}{ Entorno profesional } \\
\hline $\begin{array}{l}\text { Conocer cómo la tecnología } \\
\text { influye en las relaciones laborales }\end{array}$ & $\begin{array}{l}\text { Desde una perspectiva sociológica crítica, se entrevistó a } \\
\text { trabajadores. }\end{array}$ \\
\hline $\begin{array}{l}\text { Lograr que los pacientes sigan los } \\
\text { tratamientos médicos }\end{array}$ & $\begin{array}{l}\text { Análisis conversacional desde modelo biopsicológico que } \\
\text { conceptualiza al individuo en términos relacionales. }\end{array}$ \\
\hline $\begin{array}{l}\text { Analizar el funcionamiento de la } \\
\text { comunicación organizacional }\end{array}$ & $\begin{array}{c}\text { Desde la Teoría de la Estructuración, que entiende que los } \\
\text { actores sociales generan la estructura, se realizaron } \\
\text { entrevistas a integrantes de organizaciones. }\end{array}$ \\
\hline $\begin{array}{l}\text { Evaluar programas para la } \\
\text { participación de trabajadores }\end{array}$ & $\begin{array}{c}\text { Desde la perspectiva de Red, entendiendo a los participantes } \\
\text { como vínculos y no como individuos, se plantearon "círculos } \\
\text { de calidad", integrados por trabajadores para ayudar a } \\
\text { compañeros. Se hicieron entrevistas estructuradas a estos } \\
\text { trabajadores para identificar cambios introducidos por los } \\
\text { círculos en el funcionamiento de la estructura en } \\
\text { organizaciones. }\end{array}$ \\
\hline $\begin{array}{c}\text { Analizar prácticas sociales en las } \\
\text { conferencias académicas }\end{array}$ & Técnica de docudrama desde una perspectiva etnográfica. \\
\hline
\end{tabular}

Fuente: Gómez-Diago (2020)

\subsection{RELACIÓN ENTRE PERSONAS Y MEDIOS DE COMUNICACIÓN}

La relación entre las personas y los medios de comunicación sigue siendo transcendente en un momento en el que las facilidades para generar y publicar contenidos en el entorno digital vuelven más necesario que nunca un periodismo vigilante con vocación

\footnotetext{
${ }^{5}$ Estructuras cognitivas que organizan secuencias apropiadas en una situación conversacional para lograr unos propósitos determinados.
} 
de servicio público, orientado a proporcionar herramientas a los ciudadanos que les sirvan para vivir mejor.

Los objetivos, las perspectivas teóricas y los métodos de investigación que se sitúan en esta categoría se ordenan en dos subcategorías: 1) Efectos de los medios de comunicación, procesamiento, recepción y usos de información y 2) Análisis del diseño de productos comunicativos.

\subsubsection{Efectos de los medios, procesamiento, recepción y usos de información}

El análisis de los efectos de los medios de comunicación es de interés para Cantor (1989) que, con el objetivo de predecir qué estímulos mediáticos provocarían reacciones problemáticas en los niños, e interesado en descubrir métodos para mitigarlas y prevenirlas, desarrollando estrategias y guías para profesores, padres y niños, hizo experimentos $^{6}$ para comprobar si sus respuestas variaban en función de su edad. El investigador, consciente de lo artificial de poner a niños a ver la televisión para analizar su comportamiento, utilizó cuestionarios abiertos para completar el trabajo.

Las maneras en las que las personas procesan información interesaron a Graber (1989) que, basándose en la Schema Theory, hizo análisis de contenido de grabaciones en las que preguntaba a personas acerca de su estilo de vida y acerca de sus opiniones respecto de asuntos políticos.

De acuerdo con la Schema Theory, los esquemas de datos (schemadata) representan el aprendizaje social y se adquieren desde la infancia mediante la enseñanza, la observación, la imitación, a través de procesos de razonamiento y mediante experiencias personales o indirectas y ayudan a las personas a seleccionar y a procesar información, aunque al basarse en estereotipos, motivan errores de juicio que manchan la información incorporada recientemente y la ya almacenada.

\footnotetext{
${ }^{6}$ Cantor (1989) realizó los experimentos desde una perspectiva que entiende que cualquier respuesta es fruto de diferentes causas y que los experimentos sirven para comprobar si la presencia de una variable específica hace que una respuesta tenga más posibilidades de darse.
} 
Que la Schema Theory posibilite saber cómo las personas van a procesar información sugiere que las opiniones políticas pueden ser más predecibles de lo que se pensaba, necesitándose para ello, según Graber (1989) conocer los siguientes aspectos: 1) Cómo procesan las personas información política para desarrollar actitudes y opiniones; 2) La cultura política de la población que se va a examinar y 3) La información política a la que la población está siendo expuesta.

Otra cuestión fundamental referida a los efectos de los medios de comunicación es el recuerdo. Considerando que en el procesamiento de la comunicación publicitaria intervienen diversas operaciones mentales, Thorson (1989) evaluó cómo se produce el recuerdo de los productos anunciados, estudiando la influencia en este recuerdo del grado de atención, de la memoria, de la involucración con el producto o de la emoción.

Murdock (1989) subrayó la necesidad de trascender el estudio de los actos de consumo de los medios de comunicación y de analizar las estructuras que proporcionan el contexto y los recursos para la actividad de la audiencia para conocer cómo ésta genera e integra significados en el día a día. El investigador planteó que al reproducirse a través de actividades concretas de la vida diaria, las estructuras deben analizarse como formaciones históricas sujetas a modificaciones, poniendo atención en cómo se construyen a través de la acción y, recíprocamente, en cómo la acción se constituye estructuralmente.

Murdock (1989) propuso analizar la audiencia no como una masa de individuos atomizada sino como un número de formaciones socioculturales o grupos cuyos miembros comparten una orientación cultural en las maneras de decodificar mensajes. El investigador (1989) identificó discursos sobre terrorismo emitidos en televisión, distinguiendo entre discursos cerrados (jerarquizados) y discursos abiertos.

También interesada en la construcción de los discursos por los medios de comunicación, Schwichtenberg (1989) desarrolló una perspectiva feminista combinada con los estudios culturales y el análisis semiótico para analizar cómo los medios reflejan y promueven conceptos como el de belleza. 
En una línea similar, Treicher (1989), con el objetivo de influir en las políticas públicas de salud, analizó el concepto de parto desde la perspectiva de los estudios culturales feministas, profundizando en cómo este concepto fue cambiando a lo largo del tiempo e identificando sectores de la sociedad que influyeron en los significados que se le fueron asignando.

Respecto del análisis de los usos de los medios, Swanson y Babrow (1989) utilizaron la perspectiva de los Usos y Gratificaciones combinada con la Expectation Value Theory para conocer acerca del consumo de noticias en televisión por los jóvenes. Los investigadores realizaron encuestas cerradas a estudiantes de la Universidad de Illinois, evaluando aspectos como la exposición, la norma social y su conducta durante el visionado de noticias. Para profundizar en la exposición, se estimó el número de veces que los encuestados vieron programas de noticias en una cadena de televisión (CBS, $N B C, A B C$ ) durante una semana. En la norma social se profundizó preguntando a los estudiantes si consideraban que ver la televisión había sido extremadamente bueno/malo, sabio/tonto, beneficioso/ dañino. La investigación, según Swanson y Babrow (1989), ilustra los beneficios de utilizar la Expectancy Value Theory para clarificar y reforzar el modelo de Usos y Gratificaciones, al ofrecer una explicación más precisa de las decisiones de exposición.

\subsubsection{Análisis del diseño de productos comunicativos}

Los trabajos que se incluyen en este epígrafe se preocupan de los factores que afectan y que condicionan el diseño de productos comunicativos. En este sentido, a Turow (1989) le interesó estudiar cómo se generan las series estadounidenses de médicos y para ello visionó diferentes series de televisión de esta temática y realizó entrevistas semiestructuradas a personas involucradas en su creación como productores, directores, ejecutivos de cadenas televisivas y consultores médicos.

Centrada en el papel de las tecnologías en la producción audiovisual, Wasko (1989) propuso investigar acerca de la influencia de las tecnologías en la existencia de una producción independiente, de productos de mayor calidad y más espectaculares, de una multiplicidad de canales de distribución y de un mayor acceso de los consumidores a los 
recursos de comunicación. Wasko (1989) planteó que estudiar la economía política de las tecnologías es fundamental, y en este sentido subrayó la importancia de preguntarse qué cambios ocasionan las tecnologías en su producción y en su distribución, además de prestar atención a quién controla su desarrollo y al contexto estructural en el que se producen y distribuyen.

Interesada también en la tecnología y entendiendo el progreso como algo no necesariamente positivo, Daryl Slack (1989) propuso utilizar la perspectiva cultural para definir la tecnología enfatizando la relación entre objetos aceptados como tecnológicos y las fuerzas sociales en las que estos objetos toman fuerza. Así, la investigadora propuso investigar asociando los ordenadores a la ideología del progreso, a la era de la información, al clima político cambiante, al género y a la cultura posmoderna.

A continuación, se incluyen objetivos, perspectivas teóricas y métodos de investigación para investigar sobre la relación entre personas y los medios de comunicación, diferenciando entre aquellos centrados en los efectos de los medios, en el procesamiento, en la recepción y en los usos de la información y los centrados en el diseño de productos comunicativos. 
Tabla 2. Objetivos, perspectivas teóricas y métodos de investigación para investigar acerca de la relación entre personas y medios de comunicación en "Paradigm exemplars" (1989).

\begin{tabular}{|c|c|}
\hline \multicolumn{2}{|c|}{ Relaciones entre personas y medios } \\
\hline Objetivos de investigación & Perspectivas y métodos de investigación \\
\hline \multicolumn{2}{|c|}{ Efectos de los medios, procesamiento, recepción y usos de información } \\
\hline $\begin{array}{l}\text { Conocer acerca de los efectos de } \\
\text { la televisión en los niños }\end{array}$ & Cuestionarios abiertos y observación experimental. \\
\hline $\begin{array}{c}\text { Conocer acerca del } \\
\text { procesamiento de información } \\
\text { política }\end{array}$ & Análisis de contenido y encuestas desde la schema theory. \\
\hline $\begin{array}{c}\text { Indagar en la interacción entre } \\
\text { estructura y acción }\end{array}$ & $\begin{array}{c}\text { Evaluación de discursos sobre terrorismo en la } \\
\text { programación televisiva. }\end{array}$ \\
\hline $\begin{array}{l}\text { Conocer cómo se produce el } \\
\text { recuerdo de los anuncios }\end{array}$ & $\begin{array}{l}\text { Hicieron experimentos partiendo de que las personas } \\
\text { responden a sugerencias sin usar una estructura de } \\
\text { actitud consciente. De esta manera, de muchas de las } \\
\text { variables que utilizaban no eran conscientes los } \\
\text { participantes. Utilizaban variables como: estructura del } \\
\text { lenguaje, involucración con el producto, distracción en el } \\
\text { visionado o instrucciones de visionado. }\end{array}$ \\
\hline $\begin{array}{l}\text { Conocer cómo los medios } \\
\text { reflejan y promueven conceptos } \\
\text { como el de belleza }\end{array}$ & $\begin{array}{c}\text { Perspectiva feminista en combinación con Estudios } \\
\text { Culturales y análisis Semiótico orientada a identificar los } \\
\text { diferentes significados asignados a estos conceptos. }\end{array}$ \\
\hline $\begin{array}{c}\text { Conocer cómo usan los jóvenes la } \\
\text { televisión }\end{array}$ & $\begin{array}{l}\text { Desde la perspectiva de los usos y gratificaciones y la } \\
\text { expectation value theory se realizaron encuestas a jóvenes } \\
\text { para conocer cómo consumen noticias. }\end{array}$ \\
\hline \multicolumn{2}{|c|}{ Análisis del diseño de productos comunicativos } \\
\hline $\begin{array}{c}\text { Conocer cómo las instituciones } \\
\text { influyen a los creadores de ficción } \\
\text { televisiva }\end{array}$ & $\begin{array}{l}\text { Análisis de series de médicos, análisis documental, } \\
\text { entrevistas a productores, asistencia a la grabación de } \\
\text { varias series. }\end{array}$ \\
\hline $\begin{array}{l}\text { Conocer la influencia de las } \\
\text { tecnologías en la producción de } \\
\text { productos culturales }\end{array}$ & $\begin{array}{c}\text { Desde la perspectiva de la economía política, se analizó } \\
\text { cómo se decide qué nuevas tecnologías se introducen y } \\
\text { qué productos se crean con ellas. }\end{array}$ \\
\hline
\end{tabular}

Fuente: Gómez-Diago (2020)

\section{CONCLUSIONES}

El campo científico de la investigación en comunicación puede tener funciones en una sociedad que lo necesita y para (re) definir esas funciones, es necesario (re) identificar y actualizar objetivos que puede acometer, así como teorías y métodos de investigación que se pueden (re) utilizar para lograrlos. Danziger (1990), investigador que hizo metainvestigación en psicología, expresa que los datos que ocupan las páginas de los journals de ese campo científico no son hechos de la naturaleza sino artefactos construidos de acuerdo con esquemas racionales aceptados en una comunidad de investigadores. Lo mismo sucede en el campo científico de la comunicación. Los artefactos construidos, es decir, las perspectivas, los conceptos y los métodos de 
investigación han dado (y dan) forma al campo, siendo fundamental desarrollar metainvestigación de las ideas que recupere herramientas conceptuales y procedimentales a partir de las que (re) construir el campo científico de la investigación en comunicación.

Los objetivos, las perspectivas teóricas y los métodos de investigación aquí presentados son susceptibles de ser (re) utilizados y/o (re) actualizados en un contexto en el que las relaciones humanas y las relaciones de las personas con los medios de comunicación siguen articulando una sociedad en la que ya es común la comunicación entre humanos y máquinas, comunicación que debe ser abordada por la investigación en comunicación superando la concepción de Internet como un canal que ha derivado en el concepto de comunicación mediada por ordenador (Gunkel, 2012, p. 18). Es necesario entender Internet como un entorno en el que se pueden realizar diferentes acciones, entorno en el que se hace patente la función de la comunicación como contexto para la interacción (Gómez-Diago, 2017; 2018a; 2018b; 2019). En futuros trabajos, el modelo de análisis aquí utilizado se aplicará para profundizar en los objetivos, en las perspectivas teóricas y en los métodos de investigación que están motivando la investigación en la actualidad. El objetivo consiste en ir identificando perspectivas y herramientas conceptuales y procedimentales que definen y que pueden definir la investigación en comunicación.

\section{REFERENCIAS BIBLIOGRÁFICAS}

Anderson, J. A. y Middleton K., M. (2015). Epistemological Movements in Communication. An Analysis of Empirical and Rhetorical Critical Scolarship. En P. J. Gehrke y W. M Keith. A Century of Communication Studies: The Unfinished Conversation. New York: Routledge.

Baladrón-Pazos, A. J, Correyero-Ruiz, B. y Manchado-Pérez, B. (2014). Tres décadas de investigación sobre comunicación en España. Análisis de las revistas científicas de comunicación (1980-2013). Communication \& Society, 27(4), 49-71. Recuperado de https://revistas.unav.edu/index.php/communication-andsociety/article/download/35979/30518/ 
Berelson, B. (1959). The State of Communication Research. The Public Opinion Quarterly, 23(1),1-6.

Bermejo-Berros, J. (2014). Evolución de los paradigmas, metodologías y campos de la comunicación en Revista Latina de Comunicación Social durante la década 2004-2013. Revista Latina de Comunicación Social, 69, 330- 353. Recuperado de https://bit.ly/3dqsRYg

Bordieu, P., Chamboredon, J. C. y Passeron, J. C. (2002). El Oficio de Sociólogo. Presupuestos Epistemológicos. Buenos Aires: Siglo XXI Editores.

Burleson, B. (1989). The Constructivist Approach to Person-Centered Communication: Analysis of a Research Exemplar. Rethinking Communication, 2, 29-46.

Bryant, J. y Miron, D. (2004). Theory and research in mass communication, Journal of Communication, 54(4), 662-704. https://doi.org/10.1111/j.1460-2466.2004.tb02650.x

Cáceres, M. D. y Caffarel Serra, C. (1992). La investigación sobre comunicación en España. Un balance cualitativo. Telos, 32, 109-124.

Caffarel-Serra, C., Ortega-Mohedano, F. y Gaitán-Moya, J. A. (2017). "Investigación en comunicación en la universidad española en el período 2007-2014", El profesional de la información, 26(2), 218-227. https://doi.org/10.3145/epi.2017.mar.08

Cantor, J. (1989). Studying Children's Emotional Reactions to Mass Media. Rethinking Communication, 2, 47-59.

Danziger, K. (1990). Constructing the subject. Historical Origins of psychological research. Cambridge: Cambridge Universtity Press.

Daryl Slack, J. (1989). Contextualizing technology, Rethinking Communication. Rethinking Communication, 2,329-345.

Dervin, B., Grossberg, L., O ' Keefe, B. y Wartella, E. (1989). Preface. Rethinking Communication, 2, 22-23. 
Frankel, R. y Beckman, H. (1989). Conversation and Compliance with Treatment Recommendations: An Application of Micro-Interactional Analysis in Medicine. Rethinking Communication, 2, 60-74.

García Jiménez, L. (2007). Las teorías de la comunicación en España: un mapa sobre el territorio de nuestra investigación (1980-2006). Madrid: Tecnos.

Gómez-Diago, G. (2017). El papel de la investigación en comunicación ante la participación ciudadana. Propuesta para investigar entendiendo la comunicación como contexto para la interacción. En J. Herrero y C. Mateos (Eds.), Del verbo al bit. Cuadernos Artesanos de Comunicación. 2o edición ampliada (pp. 1879-1899). Recuperado de https://dialnet.unirioja.es/servlet/articulo?codigo=6071964 Gómez-Diago, G. (2018a). Paradigma para la teoría crítica en la investigación en comunicación: la comunicación como contexto para la interacción. VI Congreso Internacional de la Asociación Española de Investigación en Comunicación. Salamanca, España. Recuperado de https://cutt.ly/uhB9ylY

Gómez-Diago, G. (2018b). A paradigm for communication research: Communication as a context for interaction [Preconferencia]. The fifth annual pre-conference on HumanMachine Communication, International Communication Association. 24 de mayo. Praga. Recuperado de https://hmc2018.files.wordpress.com/2017/10/conferenceagenda.pdf

Gómez-Diago, G. (2019). A threefold approach for enabling social change: Communication as context for interaction, uneven development, and recognition. En J. Servaes (Ed.), Handbook of communication for development and social change (pp. 114). Springer. Recuperado de https://cutt.ly/IhB9sph

Goodwin, C. (1989). Turn Construction and Conversational Organization. Rethinking Communication, 2, 88-102.

Graber, D. (1989). An Information Processing Approach to Public Opinion Analysis. Rethinking Communication, 2, 103-116. 
Greene, J. (1989). Action Assembly Theory: Metatheoretical Commitments, Theoretical Propositions, and Empirical Applications. Rethinking Communication, 2, 117-128.

Gunkel, D. (2012). Communication and Artificial Intelligence: Opportunities and Challenges for the 21st Century. Communication $+1,1,1-25$. Recuperado de https://scholarworks.umass.edu/cpo/vol1/iss1/1/

Günther, E. y Domahidi, E. (2017). What Communication Scholars Write About: An Analysis of 80 Years of Research in High-Impact Journals. International Journal of Communication, 11. https://ijoc.org/index.php/ijoc/article/view/6989

Jacobs, S. y Jackson, S. (1989). Building a Model of Conversational Argument". Rethinking Communication, 2, 153-171.

Kellermann; K. y Lim, T. S. (1989). Conversational Acquaintance: The Flexibility of Routinized Behaviors. Rethinking Communication, 2, 172-187.

Krippendorff, K. (2017). Three concepts to retire. Annals of the International Communication Association, 41(1), 92-99.

https://doi.org/10.1080/23808985.2017.1291281

Lacasa-Mas, I. (2017). Die spanische Kommunikationswissenschaft auf dem Weg zu internationaler Anerkennung. Ein Abriss der Fachgeschichte, Kommunikationswissenschaft im internationalen Vergleich. Transnationale Perspektiven. Cham: Springer.

López-Escobar. E.; Martín Algarra, M. (2017). Communication teaching and research in Spain. The calm and the storm, Publizistik, 62, 83-105.

https://doi.org/10.1007/s11616-016-0306-4

McPhee, R. (1989). Organizational Communication: A Structurational Exemplar. Rethinking Communication, 2, 199-212.

Martínez-Nicolás, M. (2020). La investigación sobre comunicación en España (19852015). Contexto institucional, comunidad académica y producción científica. Revista 
Latina de Comunicación Social, 75, 383-414. https://www.doi.org/10.4185/RLCS-20201432

Martínez-Nicolás, M., Saperas, E. y Humanes, M. L. (2019). Mudanzas en la cultura científica. El nuevo contexto de la práctica investigadora sobre comunicación y sus implicaciones epistemológicas. En F. Sierra, y J. Alberich (Eds.), Epistemología de la comunicación y cultura digital: retos emergentes (pp. 23-42). Granada: Universidad de Granada. Recuperado de https://dialnet.unirioja.es/servlet/articulo?codigo=7165041 Martínez-Nicolás, M. y Saperas, E. (2016). Objetos de estudio y orientación metodológica de la reciente investigación sobre comunicación en España (2008-2014). Revista Latina de Comunicación Social, 71, 1365-1384. https://doi.org/10.4185/RLCS2016

Martínez-Nicolás, M. (2014). La investigación española sobre Comunicación de mayor visibilidad internacional. Análisis de los trabajos publicados en las revistas internacionales del Journal Citation Reports (JCR) y el SCImago Journal \& Country Rank (SJR-Scopus). En IV Congreso Internacional de la Asociación Española de Investigación de la Comunicación. Bilbao, 21-24 de enero de 2014.

Martínez-Nicolás, M. y Saperas, E. (2011). La investigación sobre Comunicación en España (1998-2007). Análisis de los artículos publicados en revistas científicas. Revista Latina de Comunicación Social, 66, 101-129. Recuperado de http://www.revistalatinacs.org/11/art/926_Vicalvaro/05_Nicolas.html

Martínez-Nicolás, M. (2007). Agitación en el campo. Nueve ideas para la Investigación sobre Comunicación Política en España. Política y Sociedad, 44(2), 209-227.

Recuperado de http://revistas.ucm.es/index.php/POSO/article/view/POSO0707230209A

Martínez-Nicolás, M. (2006). Masa (en situación) Crítica. La Investigación sobre Periodismo en España: Comunidad Científica e Intereses de Conocimiento. Anàlisi, 33, 135-170. Recuperado de http://ddd.uab.cat/pub/analisi/02112175n33/02112175n33p135.pdf 
Martínez Nicolás, M. (2001). Tendencias actuais da investigación sobre comunicación en España. Estudios de Comunicación, 0, 153-168. Recuperado de http://consellodacultura.gal/mediateca/extras/CCG_2001_Estudios-de-Comunicacionno-0-2001.pdf

Masip, P. (2011). Efecto Aneca: producción española en comunicación en el Social science citation index. Anuario ThinkEPI, 5, 206-210. Recuperado de https://recyt.fecyt.es/index.php/ThinkEPI/article/view/30505

Mosco, V. (1989). Labor in Communication Industries: A Critical Sociological Perspective. Rethinking Communication, 2, 213-225.

Murdock, G. (1989). Critical Inquiry and Audience Activity. Rethinking Communication, 2, 227-249.

Neuman, W. R. y Guggenheim, L. (2011). The evolution of media effects theory: a sixstage model of cumulative research. Communication Theory, 21(2), 169-196. https://doi.org/10.1111/j.1468-2885.2011.01381.x

Pacanowsky, M. (1989). Creating and Narrating Organizational Realities. Rethinking Communication, 2, 250-257.

Peters, J. D. (2008). Institutional Opportunities for Intellectual History in Commmunication Studies. En D. W. Park y J. Pooley (Eds.), The History of Media and Communication Research. Contested Memories. New York: Peter Lang.

Peters, J. D. (1986). Institutional sources of intellectual poverty in communication research. Communication Research, 13, 527-559.

https://doi.org/10.1177\%2F009365086013004002

Planalp, S. (1989). Relational Communication and Cognition. Rethinking Communication, 2, 269-279.

Philipsen, G. (1989). An Ethnographic Approach to Communication Studies. Rethinking Communication, 2, 258-268. 
Pooley, J. (2020). The Declining Significance of Disciplinary Memory: The Case of Communication Research. Humanities Commons. https://doi.org/10.17613/X6V4$\mathrm{RH} 74$

Pooley, J. y Schwarzenegger, C. (2017). Faulty Reception: The Institutional Roots of U.S. Communication Research's Neglect of Public Sphere Scholarship. En S. Averbeck-Liertz, (Ed.). Kommunications-wissenchaft im internationalen Vergleich. Transationale Perspektiven. Cham: Springer.

Potter, W. J., Cooper, R. y Dupagne, M. (1993). The Three Paradigms of Mass Media Research In Mainstream Communication Journals. Communication Theory, 3(4), 317335. https://doi.org/10.1111/j.1468-2885.1993.tb00077.x

Rogers, E. (1989). Relational Communication. Processes and Patterns. Rethinking Communication, 2,281- 290.

Schwichtenberg, C. (1989). The Mother Lode of Feminist Research: Congruent Paradigms in the Analysis of Beauty Culture. Rethinking Communication, 2, 291-306.

Sillars, A. L. (1989). Communication, Uncertainty, and Understanding in Marriage. Rethinking Communication, 2, 307-328.

Soriano, J. (2017). La política estatal de recompenses als investigadors en comunicació: breu anàlisi crítica. Comunicació: Revista de Recerca i d'Anàlisi, 34(2), 33-51. DOI: $10.2436 / 20.3008 .01 .159$

Stohl, C. (1989). Understanding Quality Circles: A Communication Network Perspective. Rethinking Communication, 2, 346-360.

Surlin, S. H. (1991). Rethinking Communication: Vol.1. Paradigm Issues. Vol.2. Paradigm Exemplars. Canadian Journal of Communication, 16(3). Recuperado de https://www.cjc-online.ca/index.php/journal/article/view/642/548

Swanson, D. L. y Babrow, A. (1989). Uses and Gratifications: The Influence of Gratification-Seeking and Expectancy. Rethinking Communication, 2, 361- 376. 
Thorson, E. (1989). Processing Television Commercials. Rethinking Communication, 2, 397-410.

Tracy, K. (1989). Conversational Dilemmas and the Naturalistic Experiment. Rethinking Communication, 2, 411-423.

Treichler, P. (1989). What definitions do: childbirth, cultural crisis, and the challenge to Medical Discourse. Rethinking Communication, 2, 424-453.

Turow, J. (1989). Television and Institutional Power: The case of Medicine. Rethinking Communication, 2, 457-473.

Wasko J. (1989). What 's so "New" About the "New" Technologies in Hollywood? An Example of the Study of Political Economy of Communications. Rethinking Communication, 2, 474-485.

Yum, J. O. (1989). The Communication Network. Paradigm and Intercultural Communication. Rethinking Communication, 2, 487-496 